\title{
Influence du chlorpyriphos-ethyl sur quelques paramètres de reproduction chez le rat mâle albinos exposé pendant le développement post-natal
}

\author{
Augustave KENFACK ${ }^{1 *}$, Pierre WATCHO ${ }^{2}$, Ferdinand NGOULA ${ }^{1}$, Joseph \\ TCHOUMBOUÉ $^{1}$ et Pierre KAMTCHOUING ${ }^{3}$ \\ ${ }^{1}$ Département des Productions Animales, Université de Dschang, Cameroun. \\ ${ }^{2}$ Département de Biologie Animale, Université de Dschang, Cameroun. \\ ${ }^{3}$ Département de Biologie et Physiologie Animales, Université de Yaoundé I, Cameroun. \\ *Auteur correspondant, Tel: 75105666,E-mail: augustavekenfack@yahoo.fr
}

\section{RESUME}

Les effets du chlorpyriphos-ethyl (CE) sur le poids des organes reproducteurs, l'histopathologie des testicules, les caractéristiques des spermatozoïdes épididymaires et sur les performances de reproduction ont été étudiés. A cet effet, 3 doses de CE à savoir 3,50; 5,25 et 10,50 mg.kg-1 ont été administrées par voie orale, chacune quotidiennement à 6 animaux prépubères (30 jours) pendant 90 jours. Les animaux du lot témoin ont reçu de l'eau distillée. A l'issu de ce traitement, le poids relatif des testicules significativement $(\mathrm{P}<0,05)$ le moins élevé $\left(0,268 \pm 0,075 \mathrm{~g} / 100 \mathrm{~g}\right.$ de poids corporel) a été obtenu chez les rats traités avec $5,25 \mathrm{mg} \cdot \mathrm{kg}^{-1} \mathrm{par}$ rapport à celui des témoins $(0,437 \pm 0,066 \mathrm{~g} / 100 \mathrm{~g})$. Le poids de l'épididyme, du canal déférent, des vésicules séminales et de la prostate ont été comparables $(\mathrm{P}>0,05)$ quelle que soit la dose. De nombreuses altérations ont été observées dans la structure des testicules des rats traités par rapport à celle des témoins. Une baisse non significative $(\mathrm{P}>0,05)$ du nombre de spermatozoïdes de la queue de l'épididyme a été enregistrée avec les doses croissantes de CE. Le taux de spermatozoïdes épididymaires mobiles significativement $(\mathrm{P}<0,05)$ le moins élevé $\left(55,69 \pm 22,47 \%\right.$ contre $74,333 \pm 8,223 \%$ pour le control) a été obtenu chez les rats gavés avec $10,50 \mathrm{mg} \cdot \mathrm{kg}^{-1}$ de CE. Toutefois, les performances de la reproduction n’ont présenté aucune différence significative $(\mathrm{P}>0,05)$ entre les traitements.

(C) 2007 International Formulae Group. All rights reserved.

Mots clés: Pesticide, organes génitaux mâles, poids, histopathologie, qualité des spermatozoïdes, performances de reproduction.

\section{INTRODUCTION}

A cause des besoins alimentaires sans cesse croissants de la population, l'intensification de l'agriculture est à nos jours une nécessité. Elle est caractérisée par une forte utilisation des intrants chimiques dont les pesticides. Les plus sollicités sont de la famille chimique des organophosphorés (Ops). Ils sont dans l'ensemble utilisés pour la protection des plantes en culture et des denrées stockées. Le chlorpyriphos (cps) et ses dérivés sont des Ops présentant l'avantage d'être efficaces contre un nombre élevé de déprédateurs des végétaux (Cox, 1994). Ils font partie des insecticides les plus utilisés au monde (Aldridge et al., 2005 ; Padilla et al., 2005), raison pour laquelle ils ont fait l'objet de très nombreuses études de toxicité. D'après Gibson et al. (1998), aucun autre pesticide n'a bénéficié d'autant d'attention. Cependant, l'essentiel de ces études a porté sur la neurotoxicité, précisément le développement du cerveau (Qiao et al., 2003), la neurochimie (Meyer et al., 2003 ; Padilla et al., 2005 ; Richardson et Chambers, 2005), la régulation de l'expression et du fonctionnement des récepteurs du système cholinergique (Lein et Fryer , 2004 ; Smulder et al., 2004 ; Padilla et al., 2005). Quelques auteurs ont étudié les effets de ce pesticide sur la reproduction. Des 
femmes exposées au Dursban (cps) pendant la gestation ont donné naissance à des enfants avec des malformations de la tête, de la face, des yeux et des organes génitaux (Cox, 1994). Des défectuosités similaires ont été reproduites chez des animaux de laboratoire exposés à cette même formulation (Sherman, 1995). Chez la souris CF-1, l'administration orale de 25 mg.kg-1 de cps du 6 au $15^{\text {ème }}$ jour de gestation a conduit à une foetotoxicité traduite par un poids réduit à la naissance (Deacon et al., 1980).

Le chlorpyriphos-ethyl (CE) est l'un des dérivés les plus récents du cps. Malgré l'importance de son utilisation, ses effets sur la reproduction sont encore peu abordés dans la littérature. Chez la femme, Sebe et al. (2005) ont rapporté un cas d'intoxication par le CE traduite par la mort du foetus à la $19^{\text {ème }}$ semaine de gestation. Chez le rat albinos adulte gavé avec $10,50 \mathrm{mg} \cdot \mathrm{kg}^{-1}$ jour $^{-1}$ pendant 90 jours, une diminution du poids des organes génitaux, de la concentration et de la mobilité des spermatozoïdes de la queue d'épididyme a été mise en évidence dans l'une de nos études antérieures. Les effets du CE sur le système reproducteur pendant le développement néonatal, à notre connaissance ne sont pas documentés.

Dans le but de contribuer à la connaissance de l'étendue des dommages pouvant être dus au chlorpiryphos-ethyl, nous avons entrepris de rechercher ses effets sur le poids des organes génitaux, la structure histologique des testicules, la concentration et la motilité des spermatozoïdes épididymaires et les performances de la reproduction chez le rat blanc albinos exposé à partir de 30 jours après la naissance.

\section{MATERIEL ET METHODES \\ Animaux de l'étude}

Dans cette étude, 72 rats blancs albinos de souche Wistar, issus des mise-bas synchrones dont 24 mâles âgés de 28 à 32 jours et pesant entre 28 et $32 \mathrm{~g}$ au début de l'essai et 48 femelles nullipares et âgées de 4 mois ont été utilisés. Ils ont été élevés au laboratoire de Physiologie Animale de la Faculté d'Agronomie et des Sciences Agricoles de l'Université de Dschang (LN 5$7^{\circ}$, LE 8-12)-.

\section{Logement et alimentation}

Les rats expérimentaux ont été élevés à température ambiante $\left(23-25^{\circ} \mathrm{C}\right)$ dans des cages en vitre à fond tapissé d'une litière sèche. Leur alimentation était basée sur un mélange de $65 \%$ de maïs, $20 \%$ de soja, $10 \%$ de poisson et $5 \%$ de farine d'os. Tout au long de l'essai, les animaux ont eu un accès ad libitum à l'aliment et à l'eau vitaminée. Le milieu d'élevage était éclairé 12 heures sur 24 à la lumière du jour.

\section{Préparation des doses expérimentales de chlorpyriphos-ethyl}

Le chlorpyriphos-ethyl utilisé était contenu dans la formulation dénommée Pyrclorex. Les différentes doses expérimentales ont été $1 / 10,1 / 20$ et $1 / 30$ de la $\mathrm{DL}_{50}$ obtenue chez la souris adulte, soient respectivement 10,$50 ; 5,25$ et $3,50 \mathrm{mg} / \mathrm{kg}$ de poids vif. Elles ont été préparées par dilution de la solution mère dans de l'eau distillée.

\section{Conduite de l'essai}

Les jeunes mâles de 30 jours d'âge ont été répartis en groupes comparables de 6 animaux chacun. A chaque lot d'animaux a été attribuée au hasard une dose expérimentale de pesticide. 0,583 $\mathrm{ml}$ d'eau distillée ou de la préparation renfermant le chlorpyriphos-ethyl a été administrée par kilogramme de poids corporel. Le volume de la solution administrée était ajusté hebdomadairement. Le traitement s'est fait par voie orale tous les jours jusqu'à l'âge de 4 mois (pendant 90 jours). Au bout de ce délai, les animaux ont été sacrifiés par une surdose de chloroforme. Antérieurement au sacrifice, chaque mâle et deux femelles nullipares ont été logés ensemble pendant 15 jours (durée couvrant trois cycles ovariens chez le rat).

\section{Collecte des données \\ Poids des organes génitaux}

Au $90^{\text {ème }}$ jour de l'essai, chaque animal a été pesé, puis sacrifié. Les testicules, les épididymes, les canaux déférents, les vésicules séminales et la prostate ont également été pesés.

\section{Taux de spermatozoïdes mobiles}

Immédiatement après ouverture de l'abdomen, la queue de l'épididyme du côté droit du corps a été prélevée, pesée et découpée dans une solution de $\mathrm{NaCl}$ à $0,9 \%$. 
Une goutte de la préparation ainsi obtenue a été déposée sur une lame porte-objet, placée au microscope et observée à un grossissement de 400x. Les nombres de spermatozoïdes mobiles et immobiles ont été relevés sur un total d'au moins 100 cellules.

\section{Concentration des spermatozoïdes épididymaires}

Une goutte de la solution renfermant les spermatozoïdes immobilisés a été placée dans la chambre de la cellule de Thomas et couverte par une lame couvre-objet. L'ensemble a ensuite été placé sur la platine du microscope. La lecture s'est faite au grossissement 10x40. Le nombre de spermatozoïdes contenus dans 4 grands carrés a été compté. Le calcul de la concentration par gramme de l'épididyme s'est effectué ainsi qu'il suit: $\mathrm{C}=\mathrm{N}$ x 4 x v/m V où :

$\mathrm{N}=$ Nombre de spermatozoïdes contenus dans 4 grands carrés ;

4 (puisque l'hématimètre renferme 16 grands carrés) ;

$\mathrm{V}$ (volume de la chambre en $\left.\mathrm{mm}^{3}\right)$;

$\mathrm{v}$ (volume total de la solution);

$\mathrm{m}$ (masse de l'épididyme).

\section{Performances de reproduction}

Le nombre de petits nés, le nombre de nés vivants, le nombre de petits de chaque sexe ont été relevés à chaque mise-bas ainsi que le nombre de mâles ayant procréé.

\section{Histopathologie}

Dès les premières minutes suivant le sacrifice de l'animal, les testicules ont été prélevés et immergés dans la solution de Bouin pour leur fixation. Les pièces fixées et lavées à l'eau courante ont été baignées successivement dans l'éthanol à $80^{\circ}$, à $90^{\circ}$ et à $100^{\circ}$ pour y être déshydratées. Elles ont ensuite été clarifiées par deux bains successifs de 30 et 60 minutes dans le xylène. Les pièces sortant du xylène ont été soigneusement égouttées avant d'être enduites de paraffine. Des coupes ont été confectionnées à l'aide d'un microtome, colorées et observées au microscope photonique.

\section{Analyses statistiques}

Les résultats ont été exprimés en moyenne plus ou moins écart type, comparés par le test d'ANOVA et séparés par le test de Duncan au seuil de $5 \%$.

\section{RESULTATS}

Poids des organes génitaux

$\mathrm{Au}$ terme de 90 jours de traitement, le poids des organes génitaux (Tableau 1) tend à diminuer avec l'augmentation de la dose du chlorpyriphos-ethyl (CE). Cependant, les seules différences significatives $(\mathrm{P}<0,05)$ ont été observées dans les lots traités avec respectivement 5,25 et $10,50 \mathrm{mg} \mathrm{kg}^{-1}$ où le poids des testicules a été moins élevé par rapport à celui des témoins.

\section{Histopathologie des testicules}

Les photographies A, B, C et D (Figure 1) illustrent la structure histologique des testicules des rats qui ont reçu respectivement de l'eau distillée, 3,50 ; 5,25 et 10,50 mg. $\mathrm{Kg}^{-1}$. de CE.

L'examen des coupes de testicules des rats témoins montre une structure normale du testicule. De l'analyse des coupes de testicules des rats traités avec $3,50,5,25$ et $10,50 \mathrm{mg} / \mathrm{kg}$, les altérations suivantes ont été relevées: élargissement de l'espace interstitiel, vacuolisation des cellules de l'épithélium germinal, diminution du nombre ou absence des cellules de la lignée germinale (plus manifeste dans le compartiment ad-luminal) et diminution du calibre des tubes avec à certains endroits le rétrécissement de la lumière.

\section{Caractéristiques des spermatozoïdes épididymaires et performances de reproduction}

Les nombres de spermatozoïdes par queue et par unité de masse d'épididyme (Tableau 2) ont baissé non significativement $(\mathrm{P}>0,05)$ avec l'augmentation de la dose de CE. Toutefois, le taux de spermatozoïdes mobiles a été significativement $(\mathrm{P}<0,05)$ moins élevé chez les animaux ayant reçu 10,50 mg.kg-1 comparé à celui enregistré chez les témoins.

A l'issue des croisements avec les mâles expérimentaux, le pourcentage de femelles ayant mis-bas, la taille de la portée, le sex-ratio (mâle/femelle) et le taux de survie à la naissance (Tableau 3) ont été comparables $(\mathrm{P}>0,05)$ quelle que soit la dose administrée. 

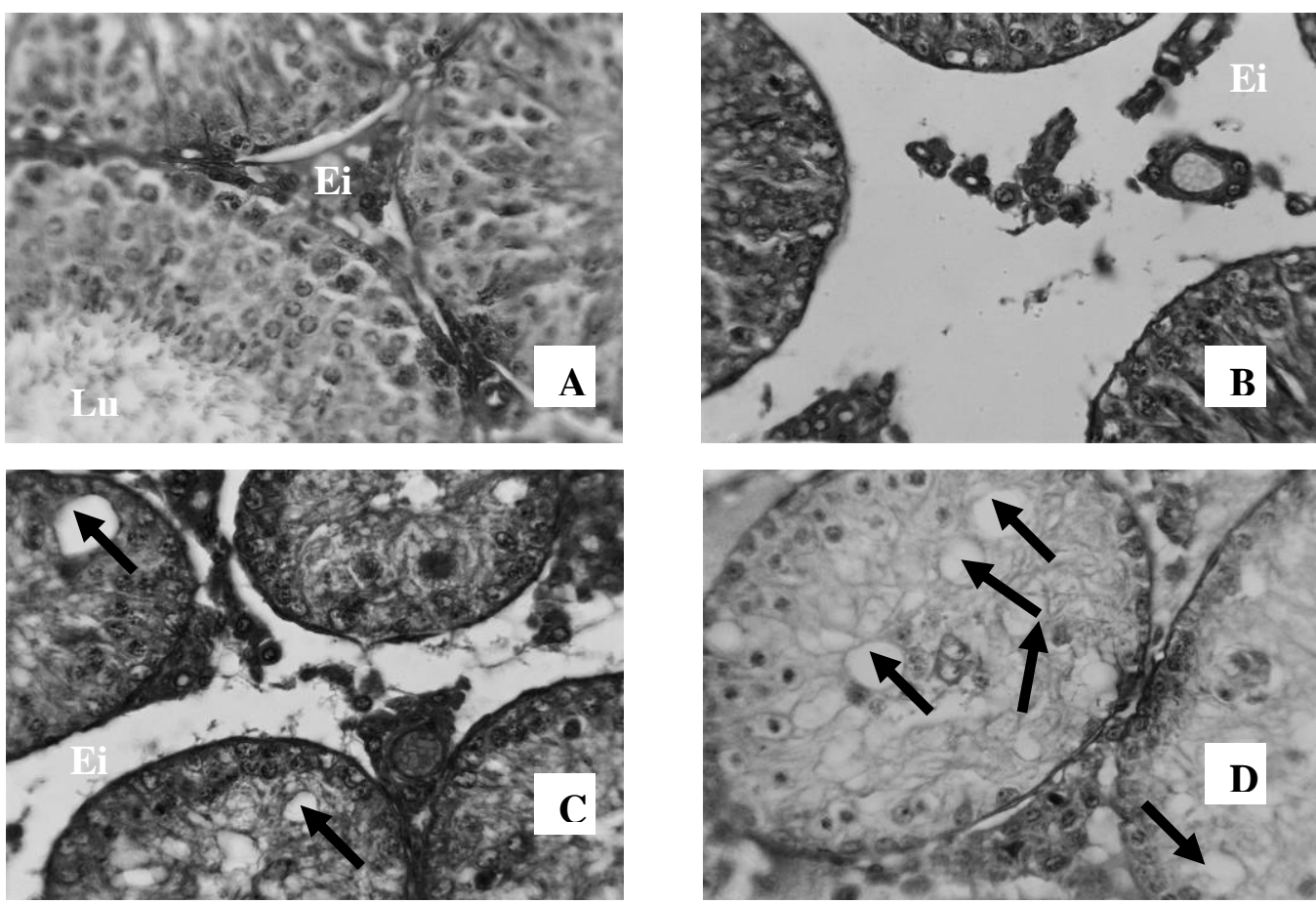

Figure 1: Structure histologique des testicules des rats témoins (photographie A) et traités avec 3,50 mg. $\mathrm{kg}^{-1}$ (photographie B), 5,25 mg. $\mathrm{kg}^{-1}$ (photographie C) et 10,50 mg. $\mathrm{kg}^{-1}$ (photographie D) de chlorpiryphos-ethyl. Ei: Espace interstitielle, Lu: Lumière du tube séminifère, $\longrightarrow$ Vacuoles. Agrandissement 400x.

Tableau 1: Effets du chlorpyriphos-ethyl sur le poids (g/100g de poids corporel) des organes génitaux chez le rat mâle.

\begin{tabular}{lcccc}
\hline \multirow{2}{*}{ Organes } & \multicolumn{4}{c}{ Doses du CE (mg.kg $\mathbf{~ j o u r ~}^{-\mathbf{1}}$ ) } \\
\cline { 2 - 5 } & 0,00 & 3,50 & 5,25 & 10,50 \\
$(\mathrm{n}=6)$ & $(\mathrm{n}=6)$ & $0,268 \pm 0,075^{\mathrm{b}}$ & $0,298 \pm 0,029^{\mathrm{b}}$ \\
& $0,437 \pm 0,066^{\mathrm{a}}$ & $0,341 \pm 0,098^{\mathrm{ab}}$ & $0,122 \pm 0,019$ \\
Testicule & $0,132 \pm 0,019$ & $0,125 \pm 0,034$ & $0,128 \pm 0,016$ & $0,122 \pm 0,035 \pm 0,05$ \\
Epididyme & $0,037 \pm 0,005$ & $0,038 \pm 0,010$ & $0,040 \pm 0,000$ & 0,035 \\
Canal déférent & $0,270 \pm 0,121$ & $0,390 \pm 0,054$ & $0,380 \pm 0,079$ & $0,323 \pm 0,130$ \\
Vésicules séminales & $0,106 \pm 0,034$ & $0,095 \pm 0,031$ & $0,098 \pm 0,022$ & $0,080 \pm 0,043$ \\
Prostate & &
\end{tabular}

a,b : dans la même ligne, les valeurs affectées de la même lettre ne diffèrent pas significativement $(\mathrm{P}>0,05)$. $\mathrm{n}:$ nombre d’observations.

Tableau 2: Influence du chlorpyriphos-ethyl sur la concentration et le taux de mobilité des spermatozoïdes épididymaires.

\begin{tabular}{lcccc}
\hline $\begin{array}{l}\text { Caractéristiques } \\
\text { spermatozoïdes }\end{array}$ & des & \multicolumn{4}{c}{ Doses du CE (mg.kg-1 jour $^{-\mathbf{1}}$ ) } \\
\cline { 2 - 5 } & \multicolumn{1}{c}{$\begin{array}{c}0,00 \\
(\mathrm{n}=6)\end{array}$} & $\begin{array}{c}3,50 \\
(\mathrm{n}=6)\end{array}$ & $\begin{array}{c}5,25 \\
(\mathrm{n}=6)\end{array}$ & $\begin{array}{c}10,50 \\
(\mathrm{n}=6)\end{array}$ \\
\hline Nombre/queue $\left(\times 10^{6}\right)$ & $15,368 \pm 4,543$ & $13,532 \pm 9,109$ & $10,902 \pm 9,668$ & $7,257 \pm 7,351$ \\
Nombre/gramme $\left(\mathrm{x} 10^{6}\right)$ & $117,297 \pm 36,973$ & $135,587 \pm 77,844$ & $104,108 \pm 89,877$ & $67,398 \pm 64,218$ \\
Mobilité $(\%)$ & $74,333 \pm 8,223^{\mathrm{a}}$ & $74,860 \pm 1,179^{\mathrm{a}}$ & $66,708 \pm 8,83^{\text {ab }}$ & $55,698 \pm 22,465^{\mathrm{b}}$ \\
\hline
\end{tabular}

a,b: dans la même ligne, les valeurs affectées de la même lettre ne diffèrent pas significativement $(\mathrm{P}>0,05)$. $\mathrm{n}: \mathrm{nombre}$ d'observations. 
Tableau 3: Influence du chlorpyriphos-ethyl sur les performances de la reproduction

\begin{tabular}{llccc}
\hline \multirow{2}{*}{ Paramètres } & \multicolumn{4}{c}{ Doses du CE (mg.kg $\left.\mathbf{~ j o u r ~}^{-\mathbf{1}}\right)$} \\
\cline { 2 - 5 } & \multicolumn{1}{c}{$\begin{array}{c}0,00 \\
(\mathrm{n}=6)\end{array}$} & $\begin{array}{c}3,50 \\
(\mathrm{n}=6)\end{array}$ & $\begin{array}{c}5,25 \\
(\mathrm{n}=6)\end{array}$ & $\begin{array}{c}10,50 \\
(\mathrm{n}=6)\end{array}$ \\
\hline Taux de fertilité $(\%)$ & $100,000 \pm 0,000^{\mathrm{a}}$ & $83,333 \pm 0,408^{\mathrm{a}}$ & $100,000 \pm 0,000^{\mathrm{a}}$ & $50,000 \pm 0,000^{\mathrm{a}}$ \\
Taille de la portée & $7,250 \pm 3,202^{\mathrm{a}}$ & $5,200 \pm 3,114^{\mathrm{a}}$ & $4,000 \pm 2,000^{\mathrm{a}}$ & $6,000 \pm 0,707^{\mathrm{a}}$ \\
Viabilité (\%) $^{\text {Sex-ratio (\%) }}$ & $85,000 \pm 23,805^{\mathrm{a}}$ & $57,500 \pm 52,736^{\mathrm{a}}$ & $100,000 \pm 0,000^{\mathrm{a}}$ & $100,000 \pm 0,000^{\mathrm{a}}$ \\
\hline
\end{tabular}

a: dans la même ligne, les valeurs affectées de la même lettre ne diffèrent pas significativement $(\mathrm{P}>0,05)$.

n: nombre d'observations.

\section{DISCUSSION}

La diminution du poids des testicules chez les rats gavés avec les fortes doses de chlorpyriphos-ethyl (CE) rappelle nos précédents résultats obtenus chez les adultes de même souche. Elle pourrait être liée à la consommation alimentaire, étant donné que les sujets insuffisamment nourris au cours de la période pré-pubertaire ne présentent pas un développement normal des gonades (Dérivaux et Ectors, 1986). En effet, le poids du testicule a baissé parallèlement à la consommation alimentaire au cours de la présente étude. Puisque les tubes séminifères représentent une part importante de la gonade mâle chez les mammifères (Dérivaux et Ectors, 1986 ; Nicolino et Forest, 2001), la diminution du poids des testicules pourrait également s'expliquer par l'atrophie de ces tubes mise en évidence sur les coupes histologiques. La relation entre le tube séminifère et la nutrition reste toutefois imprécise.

La baisse du poids de l'épididyme, des vésicules séminales et de la prostate avec les doses croissantes de CE, bien que non significative, est conforme à la conclusion de Prashanthi et al. (2006) relative au methyl parathion. Il est connu que le développement des glandes sexuelles annexes et des voies génitales extra-testiculaires est sous la dépendance des sécrétions testiculaires (Dacheux et Dacheux, 2001). C'est éventuellement la raison pour laquelle le poids de ces organes a baissé dans le même sens que celui du testicule. Un lien similaire peut être établi à l'examen des résultats des recherches réalisées par Khanna (1982), McIntyre et al. (2001), Ohsako et al. (2002) et Turner et al. (2002). La baisse de la concentration de l'épididyme en spermatozoïdes pourrait aussi être la cause de la diminution de son poids comme l'ont expliqué Klinefelter et al. (2002).

La diminution de la concentration des spermatozoïdes épididymaires et de leur taux de mobilité avec les doses croissantes de CE se rapproche des résultats obtenus par Chapin et al. (1984) à l'issue d'une exposition des rats F344 adultes au Dimethyl methylphosphanate, un pesticide organophosphoré. Par ailleurs, l'injection en dose unique de $240 \mathrm{mg} / \mathrm{kg}$ de Malathion, un autre organophosphoré a conduit après 24 heures aux résultats semblables chez la souris (Bustos-Obregon et Gonzalez-Hormazabal, 2003).

L'atteinte testiculaire caractérisée par l'analyse des coupes histologiques dans tous les lots de rats traités avec le CE conforte le résultat obtenu par Dunnick et al. (1984) chez les rats ayant reçu du Dimethyl methyl phosphanate. La présence des vacuoles dans le compartiment ad-luminal de l'épithélium germinal montre que les cellules gamétogénétiques aux stades avancés de la différentiation sont des cibles privilégiées du chlorpiryphos-ethyl. Elle apparaît comme l'explication la plus plausible de la diminution de la concentration des spermatozoïdes dans la queue d'épididyme et du diamètre des tubes séminifères telle que observée sur les photographies des coupes histologiques.

Les performances de reproduction n'ont pas été affectées par le CE. D’après Amann (1986), l'éjaculât normal de mâle renferme un grand nombre de spermatozoïdes en excès; une réduction jusqu'à $90 \%$ de la quantité de spermatozoïdes éjaculés n'altère pas sensiblement la fertilité du mâle. L'altération de la qualité de la semence due au chlorpiryphos-ethyl n'aurait pas été suffisante pour influer sur les performances de reproduction. Toutefois, le résultat de la présente étude est contraire à ceux obtenus par 
Chapin et al. (1984) et Narayana et al. (2005) qui ont rapporté des cas de sub-fertilité faisant suite à la baisse de la concentration des spermatozoïdes chez les rats adultes traités respectivement par le Dimethyl methyl phosphonate et le methyl parathion, deux pesticides organophosphorés. Huynh et al. (2000), Klinefelter et al. (2002) et Nwafia et al. (2006) ont également observé une baisse du taux de fertilité des mâles ayant présenté une diminution de la qualité du sperme, consécutive à leur exposition aux substances environnementales. Il convient néanmoins de préciser dans le cadre de la présente étude que la baisse de la concentration des spermatozoïdes avec la dose de CE n'a pas été significative.

\section{Conclusion}

L'administration du chlorpyriphosethyl chez le rat à partir de 30 jours d'âge a entraîné de nombreuses altérations dans la structure du testicule, la diminution du poids des organes génitaux, du nombre et du taux de mobilité des spermatozoïdes de la queue de l'épididyme. Cependant, les performances de la reproduction évaluées n'ont pas semblé influencées par cet insecticide.

\section{BIBLIOGRAPHIE}

Aldridge JE, Levin ED, Seidler FJ, Slotkin TA. 2005. Developmental exposure of rats to chlorpyrifos leads to behavioral alterations in adulthood, involving serotonergic mechanisms and ressembling animal model of depression. Environmental Health Perspectives, 113(5): 527-531.

Amann RP. 1986. Detection of alterations in testicular and epididymal function in laboratory animals. Environmental Health Perspectives, 70: 149-58.

Bustos-Obregon E, Gonzalez-Hormazabal P. 2003. Effects of a single dose of malathion on spermatogenesis in mice. Asian J. Androl., 5: 105-107.

Chapin RE, Dutton SL, Ross MD, Sumrell BM, Lamb JC. 1984. Development and reproductive tract lesions in male F344 rats after treatment with dimethyl methylphosphanate. Exp. Mol. Pathol., 41(1): 126-40.
Cox C. 1994. Chlorpyripfos, part 2: Human exposure. Journal of pesticide reform, 15(1): 14-28.

Dacheux F, Dacheux JL. 2001. L’épididyme et les glandes annexes. In $L a$ reproduction chez les mammifères domestiques et chez l'homme, Thibault $\mathrm{C}$, Levasseur MC (eds). INRA Editions; 290-315.

Deacon MM, Murray JS, Pilny MK, Rao KS, Dittenber DA, Hanley TR, Jr. John JA. 1980. Embryotoxicity and fetotoxicity of orally administered chlorpyriphos in mice. Toxicology and Applied Pharmacology, 54(1): 31-40.

Derivaux J, Ectors FO. 1986. Reproduction chez les mammifères domestiques. Cabay: Louvain-la-Neuve.

Dunnick JK, Gupta BN, Harris MW, Lamb JC. 1984. Reproductive toxicity of dimethyl methyl phosphanate (DMMP) in the male Fischer 344 rat. Toxicology and Applied Pharmacology, 72(3): 379-387.

Gibson JE, Peterson RKD, Shurdut BA. 1998. Human exposure and risk from indoor use of chlorpyrifos. Environmental Health Perspectives, 106(6): 303-306.

Huynh PN, Hikim AP, Wang C, Stefonovic K, Lue YH, Leung A, Atienza V, Baravarian S, Reutrakul V, Swerdloff RS. 2000. Long term effects of triptolide on spermatogenesis, epididymal sperm function and fertility in male rats. $J$. Androl., 21(5): 689-699.

Khanna HD. 1982. Biochemical alteration in male accessory sex glands after administration of alkylating agent Busulphan. Ind. J. Pharmac., 14(3): 281286.

Klinefelter GR, Srader LF, Suarez JD, Roberts NL. 2002. Bromoacetic acid exerts qualitative effects on rat sperm: implication for a novel biomarker. Toxicological Sciences, 68: 164-173.

Lein PJ, Fryer AD. 2005. Organophosphorous insecticides induce airway hyperreactivity by decreasing neuronal M2 muscarinic receptor function independent of acethylcholinesterase inhibition. Toxicological Sciences, 83: 166-176.

McIntyre BS, Barlow NJ, Foster PMD. 2001. Androgen-mediated development in male rat offspring exposed to Flutamide in utero: permanence and correlation of 
early postnatal changes in anogenital distance and nipple retention with malformation in androgen dependant tissues. Toxicological Sciences, 62: 236249.

Meyer A, Seidler FJ, Cousins MM, Slotkin TA. 2003. Developmental neurotoxicity elicited by gestational exposure to chlorpyriphos: when is Adenyl Cyclase a target? Environmental Health Perspective, 116(16).

Naravana K., Prashanthi N., Nayanatara A., Kumar HH., Abhilash K., Bairy KL. 2005. Effects of methyl parathion (0,0dimethyl 0-4-nitrophenylphosphorothioate) on rat sperm morphology and sperm count, but not fertility, are associated with decreased ascorbic acid level in the testis. Muta. Res., 7(1): 28-34.

Nicolino M, Forest MG. 2001. La puberté. In $L a$ reproduction chez les mammifères domestiques et chez l'homme. Thibault C, Levasseur MC (eds). INRA Editions; 655-679.

Nwafia WC, Igweh JC, Udebuani IN. 2006. Semen analysis of infertile Igbo males in Inugu, Eastern Nigeria. Nigerian Journal of Physiological Sciences, 21(1-2): 67-70.

Ohsako S, Miyabara Y, Sakaue M, Ishimura R, Kakeyama M, Izumi H, Yonemoto J, Tohyama C. 2002. Developmental stage specicfic effects of perinatal 2,3,7,8tetrachlorodibenzo-p-dioxin exposure on reproductive organs of male rat offspring. Toxicological Sciences, 66(2): 283-292.

Prashanthi N, Narayana K, Nayanatara A, Chandra Kumar HH, Bairy KL, D’souza UJ. 2006. The reproductive toxicity of the organophosphate pesticide 0 , 0 -dimethyl 0-4-nitrophenyl phosphorothioate (methyl parathion) in the male rat. Folia Morphol. (Warsz), 65(4): 309-21.
Padilla S, Marshall RS, Hunter DL, Oxendine S, Moser VC, Southerland SB, Mailman RB. 2005. Neurochemical effects of chronic dietary and repeated high-level acute exposure to chlorpyrifos in rats. Toxicological Sciences, 88 (1): 161-171.

Qiao D, Seidler FJ, Tate CA, Cousins MM, Slotkin TA. 2003. Fetal chlorpyrifos exposure: adverse effects on brain cell development and cholinergic biomarkers emerge postnatally and continue into adolescence and adulthood. Environmental Health Perspectives, 111(4): 536-544.

Richardson JR, Chambers JE. 2004 Neurochemical effects of repeated gestational exposure to chlorpyrifos in developing rats. Toxicological Sciences, 77: 83-90.

Sebe A, Salim Star MD, Rana Alpay MD, Nalan Kozaci MD, Ahmet Hilal MD. 2005. Organophosphate poisoning associated with foetal death: A case study. The mount Sinai Journal of medicine, 72(5).

Sherman J. 1995. Chlorpyrifos (Dursban) associated birth defects. International Journal of Occupational Medicine and Toxicology, 4(4): 417-431.

Smulder CJGM, Bueters TJH, Vailati S, Van Kleef RGDM, Vijverberg HPM. 2004. Block of neuronal nicotinic acethylcholine receptors by organophosphate insecticides. Toxicological Sciences, 82: 545-554.

Turner KJ, Barlow NJ, Struve MF, Wallace DG, Gaido KW, Dorman DC, Foster PM. 2002. Effects of in utero exposure to the organophosphate insecticide fenitrithion on androgen-dependant reproductive development in the $\mathrm{Crl}: \mathrm{CD}(\mathrm{SD}) \mathrm{BR}$ rat. Toxicological Sciences, 68: 174-183. 